\title{
AN EXCESS THEOREM FOR SPHERICAL 2-DESIGNS
}

\author{
HIROTAKE KURIHARA
}

\begin{abstract}
We give an excess theorem for spherical 2-designs. This theorem is a dual version of the spectral excess theorem for graphs, which gives a characterization of distance-regular graphs, among regular graphs in terms of the eigenvalues and the excess. Here we give a characterization of $Q$-polynomial association schemes among spherical 2-designs.
\end{abstract}

\section{INTRODUCTION}

In graph theory, distance-regularity is considered an important concept, and there are many papers on distance-regular graphs. The reader is referred to BrouwerCohen-Neumaier [3. Especially the methods to check whether a given graph is distance-regular or not are of interest. One of these methods is the spectral excess theorem 9, 7, 10. The purpose of the present paper is to give a "dual version" of this theorem.

We briefly recall the spectral excess theorem. For the rest of this section, $\Gamma=$ $(X, E)$ denotes an undirected, simple, connected and finite graph of order $n$ and diameter $D(\Gamma)=D$. The path distance between two vertices $x$ and $y$ is represented by $\partial(x, y)$. Let $\Gamma_{i}(x)$ be the set of vertices at distance $i$ from $x$, for $0 \leq i \leq D$. The $i$-th distance matrix $A_{i}$ of $\Gamma$ is defined to be the matrix with rows and columns indexed by $X$ whose $(x, y)$-entries are

$$
\left(A_{i}\right)_{x, y}= \begin{cases}1 & \text { if } y \in \Gamma_{i}(x), \\ 0 & \text { otherwise. }\end{cases}
$$

In particular $A_{1}$ is the adjacency matrix of $\Gamma$ and denoted simply by $A$. The spectrum of $\Gamma$ is the set of distinct eigenvalues of $A$ together with their multiplicities, and it will be denoted by $\operatorname{Spec}(\Gamma)=\left\{\theta_{0}^{m_{0}}, \theta_{1}^{m_{1}}, \ldots, \theta_{d}^{m_{d}}\right\}$. Then we have $D \leq d$. Suppose $\Gamma$ is a connected $k$-regular graph. Then $k$ is an eigenvalue of $\Gamma$ with multiplicity 1 . Hence we may put $\theta_{0}=k$ and $m_{0}=1$. Let $Z(t)=\prod_{i=0}^{d}\left(t-\theta_{i}\right) \in$ $\mathbb{R}[t]$, and we consider an inner product on $\mathbb{R}[t] /(Z)$ by, for $p, q \in \mathbb{R}[t] /(Z)$,

$$
\langle p, q\rangle=\frac{1}{n} \sum_{i=0}^{d} m_{i} p\left(\theta_{i}\right) q\left(\theta_{i}\right) .
$$

The predistance polynomials $p_{0}, p_{1}, \ldots, p_{d}$ of $\Gamma$ are the unique polynomials satisfying $\operatorname{deg} p_{i}=i$ and $\left\langle p_{i}, p_{j}\right\rangle=\delta_{i, j} p_{i}\left(\theta_{0}\right)$ for $i, j \in\{0,1, \ldots, d\}$, where $\delta_{i, j}$ denotes Kronecker's delta function. The graph $\Gamma=(X, E)$ is called distance-regular if $D=d$, and $A_{i}=p_{i}(A)$ for each $i \in\{0,1, \ldots, d\}$.

Date: 15 Mar., 2012.

2010 Mathematics Subject Classification. Primary 05E30, Secondary 05B30.

Key words and phrases. Q-polynomial association scheme, distance set, spherical design.

The author is supported by JSPS Research Fellowship. 
It is well known that regularity of a graph is determined by the spectrum of the graph, cf. Cvetković-Doob-Sachs [6, Theorem 3.22]. On the other hand, distanceregularity of a graph is in general not determined only by the spectrum of the graph. The spectral excess theorem states a condition that a connected regular graph is distance-regular. Here the excess of a vertex $x$ is $\left|\Gamma_{d}(x)\right|$. This result was first proved by Fiol-Garriga 9] using a local approach. Later, Van Dam [7] and Fiol-Gago-Garriga [10] gave the excess theorem using mean excess, which used a global approach. The following is the spectral excess theorem due to Fiol-GagoGarriga [10.

Theorem 1.1. Let $\Gamma$ be a regular graph with $d+1$ distinct eigenvalues and spectrally maximum diameter $D=d$. Then $\frac{1}{n} \sum_{x \in X}\left|\Gamma_{d}(x)\right| \leq p_{d}\left(\theta_{0}\right)$. Moreover equality is attained if and only if $\Gamma$ is a distance-regular graph.

Every distance-regular graph has the structure of a $P$-polynomial association scheme. The notation and some properties about association schemes are given in Section 2. The Q-polynomial property is an algebraically dual concept of the $P$-polynomial property.

In this paper we consider an excess theorem related to $Q$-polynomial association schemes. Here, the "dual" concept of regular graphs is corresponding to properties of spherical 2-designs. In Section 3, the definition of spherical designs is given, and we consider the polynomial functions on spherical 2-designs. Our main result is given and proved in Section 4 .

\section{Association schemes}

We begin with a review of basic definitions concerning association schemes. The reader is referred to Bannai-Ito [2 for the background material.

A symmetric association scheme $\mathfrak{X}=\left(X,\left\{R_{i}\right\}_{i=0}^{d}\right)$ consists of a finite set $X$ and a set $\left\{R_{i}\right\}_{i=0}^{d}$ of non-empty binary relations on $X$ satisfying:

(1) $R_{0}=\{(x, x) \mid x \in X\}$,

(2) $\left\{R_{i}\right\}_{i=0}^{d}$ is a partition of $X \times X$,

(3) ${ }^{t} R_{i}=R_{i}$ for each $i \in\{0,1, \ldots, d\}$, where ${ }^{t} R_{i}=\left\{(y, x) \mid(x, y) \in R_{i}\right\}$,

(4) the numbers $\mid\left\{z \in X \mid(x, z) \in R_{i}\right.$ and $\left.(z, y) \in R_{j}\right\} \mid$ are constant whenever $(x, y) \in R_{k}$, for each $i, j, k \in\{0,1, \ldots, d\}$.

The numbers $\mid\left\{z \in X \mid(x, z) \in R_{i}\right.$ and $\left.(z, y) \in R_{j}\right\} \mid$ are called the intersection numbers and denoted by $p_{i, j}^{k}$. Let $M_{X}(\mathbb{R})$ denote the algebra of matrices over the real field $\mathbb{R}$ with rows and columns indexed by $X$. The $i$-th adjacency matrix $A_{i}$ in $M_{X}(\mathbb{R})$ of $\mathfrak{X}$ is defined by

$$
\left(A_{i}\right)_{x, y}= \begin{cases}1 & \text { if }(x, y) \in R_{i}, \\ 0 & \text { otherwise }\end{cases}
$$

From the definition of association schemes, it follows that

(A1) $A_{0}=I$, where $I$ is the identity matrix,

(A2) $A_{0}+A_{1}+\cdots+A_{d}=J$, where $J$ is the all-one matrix, and $A_{i} \circ A_{j}=\delta_{i, j} A_{i}$ for $i, j \in\{0,1, \ldots, d\}$, where $\circ$ denotes the Hadamard product, that is, the entry-wise matrix product,

(A3) ${ }^{t} A_{i}=A_{i}$ for each $i \in\{0,1, \ldots, d\}$,

(A4) $A_{i} A_{j}=\sum_{k=0}^{d} p_{i, j}^{k} A_{k}$, for each $i, j \in\{0,1, \ldots, d\}$. 
The vector space $\mathfrak{A}=\operatorname{Span}_{\mathbb{R}}\left\{A_{0}, A_{1}, \ldots, A_{d}\right\}$ with a basis $\left\{A_{i}\right\}_{i=0}^{d}$ forms a commutative algebra and is called the Bose-Mesner algebra of $\mathfrak{X}$. It is well known that $\mathfrak{A}$ is semi-simple, hence $\mathfrak{A}$ has a second basis $E_{0}, E_{1}, \ldots, E_{d}$ satisfying the following conditions:

(E1) $E_{0}=\frac{1}{|X|} J$

(E2) $E_{0}+E_{1}+\cdots+E_{d}=I$ and $E_{i} E_{j}=\delta_{i, j} E_{i}$,

(E3) ${ }^{t} E_{i}=E_{i}$ for each $i \in\{0,1, \ldots, d\}$,

(E4) $E_{i} \circ E_{j}=\frac{1}{|X|} \sum_{k=0}^{d} q_{i, j}^{k} E_{k}$ for some real numbers $q_{i, j}^{k}$, for each $i, j \in\{0,1, \ldots, d\}$.

Then $E_{0}, E_{1}, \ldots, E_{d}$ are the primitive idempotents of the Bose-Mesner algebra $\mathfrak{A}$. The first eigenmatrix $P=\left(P_{i}(j)\right)_{j, i=0}^{d}$ and the second eigenmatrix $Q=\left(Q_{i}(j)\right)_{j, i=0}^{d}$ of $\mathfrak{X}$ are defined by

$$
A_{i}=\sum_{j=0}^{d} P_{i}(j) E_{j} \text { and } E_{i}=\frac{1}{|X|} \sum_{j=0}^{d} Q_{i}(j) A_{j}
$$

respectively.

We call $\mathfrak{X}$ a $P$-polynomial scheme (or a metric scheme) with respect to the ordering $\left\{A_{i}\right\}_{i=0}^{d}$, if for each $i \in\{0,1, \ldots, d\}$, there exists a polynomial $v_{i}$ of degree $i$, such that $A_{i}=v_{i}\left(A_{1}\right)$. Moreover $\mathfrak{X}$ is called a $P$-polynomial scheme with respect to $A_{1}$ if it has the $P$-polynomial property with respect to some ordering $A_{0}, A_{1}, A_{i_{2}}, A_{i_{3}}, \ldots, A_{i_{d}}$. Dually, $\mathfrak{X}$ is called a $Q$-polynomial scheme (or a cometric scheme) with respect to the ordering $\left\{E_{i}\right\}_{i=0}^{d}$, if for each $i \in\{0,1, \ldots, d\}$, there exists a polynomial $v_{i}^{*}$ of degree $i$, such that $|X| E_{i}=v_{i}^{*}\left(\left(|X| E_{1}\right)^{\circ}\right)$, where, for $f \in \mathbb{R}[t]$ and $M=\left(M_{x, y}\right)_{x, y \in X} \in M_{X}(\mathbb{R})$, we define $f\left(M^{\circ}\right)=\left(f\left(M_{x, y}\right)\right)_{x, y \in X}$. Moreover $\mathfrak{X}$ is called a $Q$-polynomial scheme with respect to $E_{1}$ if it has the $Q$-polynomial property with respect to some ordering $E_{0}, E_{1}, E_{i_{2}}, E_{i_{3}}, \ldots, E_{i_{d}}$. In fact, an ordering of a $Q$-polynomial association scheme with respect to $E_{1}$ is uniquely determined (cf. Kurihara-Nozaki [13]). It is known that $v_{i}$ and $v_{i}^{*}$ form systems of orthogonal polynomials [2].

\section{Harmonic polynomials on Spherical 2-Designs and Predegree POLYNOMIALS}

In this section, we consider the decomposition of the space of functions on a spherical 2-design into the subspaces consisting of harmonic functions of the same degree. Also we consider the predegree polynomials, which are determined by the angles among the elements of the spherical 2-design.

Let $r \mathbb{S}^{m-1}=\left\{\left(x_{1}, x_{2}, \ldots, x_{m}\right) \in \mathbb{R}^{m} \mid x_{1}^{2}+x_{2}^{2}+\cdots+x_{m}^{2}=r^{2}\right\}$ be the sphere of radius $r$ centered at the origin in $\mathbb{R}^{m}$, endowed with the standard inner product $\boldsymbol{x} \cdot \boldsymbol{y}=\sum_{i=1}^{m} x_{i} y_{i}$ for $\boldsymbol{x}=\left(x_{1}, x_{2}, \ldots, x_{m}\right), \boldsymbol{y}=\left(y_{1}, y_{2}, \ldots, y_{m}\right) \in \mathbb{R}^{m}$. In particular $1 \mathbb{S}^{m-1}$ is denoted simply by $\mathbb{S}^{m-1}$. For a nonempty finite set $X$ in $r \mathbb{S}^{m-1}$, we set $A(X):=\{\boldsymbol{x} \cdot \boldsymbol{y} \mid \boldsymbol{x}, \boldsymbol{y} \in X, \boldsymbol{x} \neq \boldsymbol{y}\}$ and $A^{\prime}(X):=A(X) \cup\left\{r^{2}\right\}, X$ is called an $s$-distance set if $|A(X)|=s$.

The concept of spherical designs was introduced by Delsarte-Goethals-Seidel [8], and we refer also to Bannai-Bannai [1] and 8 for detail of spherical designs. 
Definition 3.1 (Spherical design). Let $\tau$ be a nonnegative integer. A finite nonempty subset $X$ of $r \mathbb{S}^{m-1}$ is called a spherical $\tau$-design if

$$
\frac{1}{\mu\left(r \mathbb{S}^{m-1}\right)} \int_{r \mathbb{S}^{m-1}} f(\boldsymbol{x}) d \mu(\boldsymbol{x})=\frac{1}{|X|} \sum_{\boldsymbol{x} \in X} f(\boldsymbol{x})
$$

holds for all polynomials $f(\boldsymbol{x})=f\left(x_{1}, x_{2}, \ldots, x_{m}\right)$ of degree at most $\tau$. Here $\mu$ is the Lebesgue measure on $r \mathbb{S}^{m-1}$.

Let $X$ be a nonempty finite set in $\sqrt{m} \mathbb{S}^{m-1}$ of size $n$. For $\alpha \in A^{\prime}(X)$, the relation matrix $A_{\alpha}$ is defined to be the matrix in $M_{X}(\mathbb{R})$ with

$$
\left(A_{\alpha}\right)_{\boldsymbol{x}, \boldsymbol{y}}= \begin{cases}1 & \text { if } \boldsymbol{x} \cdot \boldsymbol{y}=\alpha, \\ 0 & \text { otherwise }\end{cases}
$$

The normalized Gram matrix $G$ of $X$ is defined by $G:=\frac{1}{n} \sum_{\alpha \in A^{\prime}(X)} \alpha A_{\alpha}$. Since $X$ is in the sphere of radius $\sqrt{m}, X$ satisfies

(TD1) $\boldsymbol{x} \cdot \boldsymbol{x}=m$ for each $\boldsymbol{x} \in X$, i.e., $G \circ I=\frac{m}{n} I$,

(TD2) $\boldsymbol{x} \cdot \boldsymbol{y}<m$ for each $\boldsymbol{x}, \boldsymbol{y} \in X$ with $\boldsymbol{x} \neq \boldsymbol{y}$, i.e., $A_{m}=I$.

Moreover it is known that $X$ is a spherical 2-design if and only if

(TD3) $\sum_{\boldsymbol{y} \in X} \boldsymbol{x} \cdot \boldsymbol{y}=0$ for each $\boldsymbol{x} \in X$, i.e., $G J=0$,

(TD4) $\frac{1}{n} \sum_{\boldsymbol{z} \in X}(\boldsymbol{x} \cdot \boldsymbol{z})(\boldsymbol{z} \cdot \boldsymbol{y})=\boldsymbol{x} \cdot \boldsymbol{y}$ for each $\boldsymbol{x}, \boldsymbol{y} \in X$, i.e., $G^{2}=G$,

(cf. [8). This condition describe that the center of $X$ is the origin of $\mathbb{R}^{m}$ and $X$ is an eutectic star [5, 8.

Remark 3.2. The condition (TD1)-(TD4) is an algebraically dual condition of simple, connected and regular graphs. In fact, a graph of order $n$ is simple, $k$-regular, connected if and only if the adjacency matrix $A$ of the graph satisfies $A \circ I=0$, $A \circ A=A, A J=k J$ and $E_{0}=\frac{1}{n} J$, where $E_{0}$ is the orthogonal projection onto the eigenspace of $A$ with eigenvalue $k$. This duality is similar to the duality of the adjacency matrices and the primitive idempotents of association schemes.

For a $Q$-polynomial association scheme $\mathfrak{X}=\left(X,\left\{R_{i}\right\}_{i=0}^{s}\right)$ with respect to $E_{1}$, the mapping $x \mapsto\left(|X| E_{1}\right)_{x}$, sending each vertex $x$ to the $x$-th column of $|X| E_{1}$, determines a mapping from $X$ to a finite subset $\tilde{X}$ in $\operatorname{Span}_{\mathbb{R}}\left\{\left(|X| E_{1}\right)_{y} \mid y \in X\right\} \cong$ $\mathbb{R}^{m}$, where $m=\operatorname{rank} E_{1}$. It is known that $\tilde{X}$ can be an $s$-distance set in $\sqrt{m} \mathbb{S}^{m-1}$, and $\tilde{X}$ is always a spherical 2-design (cf. Cameron-Goethals-Seidel [4]). On the other hand, not all spherical 2-designs arise in this way. We are interested in when a spherical 2-design has the structure of a $Q$-polynomial association scheme. There exists a result for this "Q-polynomial property" of spherical designs. DelsarteGoethals-Seidel 8 . proved that a $\tau$-design $X$ which is an $s$-distance set with $\tau \geq$ $2 s-2$ has the $Q$-polynomial property. In fact the condition $\tau \geq 2 s-2$ seems too strong. In the present paper, we only focus on the $Q$-polynomial property of 2-designs.

For a finite subset $X$ in $\sqrt{m} \mathbb{S}^{m-1}$ of size $n$, the vector space of $\mathbb{R}$-valued functions on $X$ is denoted by $C(X)$. We equip $C(X)$ with an inner product by

$$
(f, g)=\frac{1}{n} \sum_{\boldsymbol{x} \in X} f(\boldsymbol{x}) g(\boldsymbol{x})
$$


for $f, g \in C(X)$. For a polynomial $p \in \mathbb{R}[t]$ and $\boldsymbol{a} \in X$, we define the zonal polynomial $\zeta_{\boldsymbol{a}}(p): X \rightarrow \mathbb{R}$ of $p$ at $\boldsymbol{a}$ by $\zeta_{\boldsymbol{a}}(p)(x)=p(\boldsymbol{a} \cdot \boldsymbol{x})$. We further define the vector spaces $\operatorname{Pol}_{k}(X)$, which first appeared in Godsil [11, recursively by setting:

- $\operatorname{Pol}_{0}(X)$ is the set of constant functions on $X$,

- $\operatorname{Pol}_{1}(X)=\operatorname{Span}_{\mathbb{R}}\left\{\zeta_{\boldsymbol{a}}(p) \mid \boldsymbol{a} \in X, \operatorname{deg} p \leq 1\right\}$,

- $\operatorname{Pol}_{k}(X)=\operatorname{Span}_{\mathbb{R}}\left\{f g \mid f \in \operatorname{Pol}_{1}(X), g \in \operatorname{Pol}_{k-1}(X)\right\}$ for $k \geq 2$.

If $f \in \operatorname{Pol}_{k}(X) \backslash \operatorname{Pol}_{k-1}(X)$, then we say that $f$ has degree $k$. From the definition of $\operatorname{Pol}_{k}(X)$, it seems complicated to describe elements of $\mathrm{Pol}_{k}(X)$, but we can simplify the description of $\mathrm{Pol}_{k}(X)$.

Lemma 3.3 (cf. Theorem 4.1 in Chapter 15 in Godsil [12]). For each nonnegative integer $k$,

$$
\operatorname{Pol}_{k}(X)=\operatorname{Span}_{\mathbb{R}}\left\{\zeta_{\boldsymbol{a}}(p) \mid \boldsymbol{a} \in X, \operatorname{deg} p \leq k\right\} .
$$

Next we define the degree of $X$.

Lemma 3.4. Suppose a finite set $X \subset \sqrt{m} \mathbb{S}^{m-1}$ is an s-distance set. Then

$$
C(X)=\mathrm{Pol}_{s}(X) \text {. }
$$

Proof. Let $F(t)=\prod_{\alpha \in A(X)} \frac{t-\alpha}{m-\alpha}$. Then by (TD1) and (TD2), for each $\boldsymbol{y} \in X$, the function $\zeta_{\boldsymbol{y}}(F)(\boldsymbol{x})$ is equal to 1 if $\boldsymbol{x}=\boldsymbol{y}$, and 0 otherwise. Namely $\left\{\zeta_{\boldsymbol{y}}(F)\right\}_{\boldsymbol{y} \in X}$ is a basis of $C(X)$. Since $\operatorname{deg} F=s$, we have $\zeta_{\boldsymbol{y}}(F) \in \operatorname{Pol}_{s}(X)$ for all $\boldsymbol{y} \in X$. Thus, $C(X)=\operatorname{Pol}_{s}(X)$, as desired.

We set $S=\min \left\{i \in\{0,1, \ldots, s\} \mid \operatorname{Pol}_{i}(X)=C(X)\right\}$, and we call $S$ the degree of $X$. By Lemma 3.4 we have $S \leq s$.

For the rest of this paper we always suppose that $X$ is a spherical 2-design of size $n$ in $\sqrt{m} \mathbb{S}^{m-1}$. Let $\operatorname{Harm}_{0}(X)=\operatorname{Pol}_{0}(X)$ and we define

$$
\operatorname{Harm}_{k}(X)=\operatorname{Pol}_{k}(X) \cap \operatorname{Pol}_{k-1}(X)^{\perp} \text { for } k \geq 1 .
$$

Its elements are harmonic polynomials of degree $k$. From the definition of the degree of $X$, we get

$$
\operatorname{Harm}_{j}(X) \neq\{0\}(0 \leq j \leq S) \text { and } C(X)=\bigoplus_{i=0}^{S} \operatorname{Harm}_{i}(X) .
$$

By (TD [3), for a polynomial $r_{1} t+r_{2} \in \mathbb{R}[t]$ of degree 1 and $\boldsymbol{a} \in X$, we have

$$
\begin{aligned}
\left(1, \zeta_{\boldsymbol{a}}\left(r_{1} t+r_{2}\right)\right) & =\frac{1}{n} \sum_{\boldsymbol{x} \in X}\left(r_{1} \boldsymbol{a} \cdot \boldsymbol{x}+r_{2}\right) \\
& =\frac{r_{1}}{n} \sum_{\boldsymbol{x} \in X} \boldsymbol{a} \cdot \boldsymbol{x}+r_{2} \\
& =r_{2},
\end{aligned}
$$

where $\mathbf{1}(\boldsymbol{x})=1$ for any $\boldsymbol{x} \in X$. Hence $\operatorname{Harm}_{1}(X)=\operatorname{Span}_{\mathbb{R}}\left\{\zeta_{\boldsymbol{a}}(t) \mid \boldsymbol{a} \in X\right\}$.

Now $M_{X}(\mathbb{R})$ acts on $C(X)$ by $(M f)(\boldsymbol{x})=\sum_{\boldsymbol{y} \in X} M_{\boldsymbol{x}, \boldsymbol{y}} f(\boldsymbol{y})$ for $M \in M_{X}(\mathbb{R})$ and $f \in C(X)$. Let $F_{i}$ be the projection matrix from $C(X)$ onto $\operatorname{Harm}_{i}(X)$, that is, $F_{i} f$ is equal to $f$ if $f \in \operatorname{Harm}_{i}(X), 0$ if $f \in \bigoplus_{j=0, j \neq i}^{S} \operatorname{Harm}_{j}(X)$. Then $\left\{F_{i}\right\}_{i=0}^{S}$ satisfy $\sum_{i=0}^{S} F_{i}=I, F_{j} \neq 0$ and $F_{j}^{2}=F_{j}$ for $j \in\{0,1, \ldots, S\}$. We call $\left\{F_{i}\right\}_{i=0}^{S}$ the projection matrices of $X$.

Lemma 3.5. $F_{0}=\frac{1}{n} J$ and $F_{1}=G$. 
Proof. Since $\left(\frac{1}{n} J \cdot 1\right)(x)=\frac{1}{n} \sum_{x \in X} 1=1(x)$ for every $x \in X$ and every $f \in$ $\operatorname{Harm}_{0}(X)^{\perp}$ satisfies $\left(\frac{1}{n} J f\right)(\boldsymbol{x})=\frac{1}{n} \sum_{\boldsymbol{x} \in X} 1 \cdot f(\boldsymbol{x})=(\mathbf{1}, f)=0$, it follows that $F_{0}=\frac{1}{n} J$.

By (TD4), we have

$$
\begin{aligned}
\left(G \zeta_{\boldsymbol{y}}(t)\right)(\boldsymbol{x}) & =\sum_{\boldsymbol{z} \in X} \frac{1}{n}(\boldsymbol{x} \cdot \boldsymbol{z})(\boldsymbol{z} \cdot \boldsymbol{y}) \\
& =\boldsymbol{y} \cdot \boldsymbol{x} \\
& =\zeta_{\boldsymbol{y}}(t)(\boldsymbol{x}) .
\end{aligned}
$$

Also, every $f \in \operatorname{Harm}_{1}(X)^{\perp}$ satisfies $(G f)(\boldsymbol{x})=\sum_{\boldsymbol{y} \in X} \frac{1}{n}(\boldsymbol{x} \cdot \boldsymbol{y}) f(\boldsymbol{y})=\left(\zeta_{\boldsymbol{x}}(t), f\right)=$ 0 . Therefore, it follows that $G=F_{1}$.

Lemma 3.6. For $j>i, F_{j} G^{\circ i}=0$.

Proof. The $\boldsymbol{y}$-th column of $G^{\circ i}$ can be regarded as the zonal polynomial $\zeta_{\boldsymbol{y}}\left(t^{i}\right)$ of $t^{i}$ at $\boldsymbol{y}$. Then $\zeta_{\boldsymbol{y}}\left(t^{i}\right) \in \operatorname{Pol}_{i}(X)=\bigoplus_{k=0}^{i} \operatorname{Harm}_{k}(X)$. Hence we get $F_{j} G^{\circ i}=0$.

Suppose $X$ is an $s$-distance set with the normalized Gram matrix $G$. For $\alpha \in$ $A^{\prime}(X)$, denote $\left|\left\{(\boldsymbol{x}, \boldsymbol{y}) \in X^{2} \mid \boldsymbol{x} \cdot \boldsymbol{y}=\alpha\right\}\right|$ by $\kappa_{\alpha}$. We put $Z^{*}(t)=\prod_{\alpha \in A^{\prime}(X)}(t-\alpha)$. We define an inner product on $\mathbb{R}[t] /\left(Z^{*}\right)$ by, for $p, q \in \mathbb{R}[t] /\left(Z^{*}\right)$,

$$
\langle p, q\rangle=\frac{1}{n^{2}} \sum_{\alpha \in A^{\prime}(X)} \kappa_{\alpha} p(\alpha) q(\alpha) .
$$

The predegree polynomials $q_{0}, q_{1}, \ldots, q_{s}$ of $X$ are the polynomials satisfying $\operatorname{deg} q_{k}=$ $k$ and $\left\langle q_{k}, q_{h}\right\rangle=\delta_{k, h} q_{k}(m)$ for any $k, h \in\{0,1, \ldots, s\}$. As a sequence of orthogonal polynomials, the predegree polynomials satisfy a three-term recurrence of the form

$$
t q_{k}=b_{k-1}^{*} q_{k-1}+a_{k}^{*} q_{k}+c_{k+1}^{*} q_{k+1} \quad(0 \leq k \leq s),
$$

where the constants $b_{k-1}^{*}, a_{k}^{*}$ and $c_{k+1}^{*}$ are the Fourier coefficients of $t q_{k}$ in terms of $\left\{q_{i}\right\}_{i=0}^{s}$ respectively (and $b_{-1}^{*}=c_{s+1}^{*}=0$ ). Moreover, the predegree polynomials satisfy the following equation, cf. [9]:

$$
\sum_{i=0}^{s} q_{i}(t)=n \prod_{\alpha \in A(X)} \frac{t-\alpha}{m-\alpha}
$$

To see this, just note that $\left\langle n \prod_{\alpha \in A(X)} \frac{t-\alpha}{m-\alpha}, q_{k}\right\rangle=\frac{1}{n} \kappa_{m} q_{k}(m)=q_{k}(m)$. We put $H(t)=\sum_{i=0}^{s} q_{i}(t)$. Then $\frac{1}{n} H\left((n G)^{\circ}\right)=I$.

Lemma 3.7. Let $\mathfrak{X}=\left(X,\left\{R_{i}\right\}_{i=0}^{s}\right)$ be a $Q$-polynomial scheme, with $|X|=n$, with respect to $E_{1}$. Then the degree $S$ of the image of the spherical embedding of $\mathfrak{X}$ with respect to $E_{1}$ is $s$. Moreover, for each $i \in\{0,1, \ldots, s\}, F_{i}=\frac{1}{n} q_{i}\left((n G)^{\circ}\right)$.

Proof. Let $\left\{E_{i}\right\}_{i=0}^{s}$ be the $Q$-polynomial ordering of $\mathfrak{X}$, and let $\left\{v_{i}^{*}\right\}_{i=0}^{s}$ be the polynomials such that $E_{i}=\frac{1}{n} v_{i}^{*}\left(\left(n E_{1}\right)^{\circ}\right)$ for each $i \in\{0,1, \ldots, s\}$. By Lemma 3.3. we obtain $\operatorname{Pol}_{i}(X)=\operatorname{Span}_{\mathbb{R}}\left\{\zeta_{a}(p) \mid a \in X, \operatorname{deg} p \leq i\right\}=\sum_{j=0}^{i}\left(E_{1}\right)^{\circ j} C(X)$. Moreover, we obtain $\sum_{j=0}^{i}\left(E_{1}\right)^{\circ j} C(X)=E_{0} C(X) \perp E_{1} C(X) \perp \cdots \perp E_{i} C(X)$. Hence $\operatorname{Harm}_{i}(X)=E_{i} C(X)$ and $F_{i}=E_{i}$ for each $i \in\{0,1, \ldots, s\}$, and thus $S=s$. 
Finally we prove $q_{i}=v_{i}^{*}$ for $i \in\{0,1, \ldots, s\}$. For $i, j \in\{0,1, \ldots, s\}$, we get

$$
\begin{aligned}
\left\langle v_{i}^{*}, v_{j}^{*}\right\rangle & =\frac{1}{n^{2}} \sum_{a, b \in X} v_{i}^{*}(a \cdot b) v_{j}^{*}(a \cdot b) \\
& =\sum_{a, b \in X}\left(E_{i}\right)_{a, b}\left(E_{j}\right)_{a, b} \\
& =\delta_{i, j} \operatorname{tr} E_{i} \\
& =\delta_{i, j} v_{i}^{*}(m),
\end{aligned}
$$

that is $\left\{v_{i}^{*}\right\}_{i=0}^{s}$ coincide with the predegree polynomials $\left\{q_{i}\right\}_{i=0}^{s}$ of $X$. Therefore we get the desired result.

Lemma 3.8. Suppose a spherical 2-design $X$ is an $s$-distance set with $S=s$. If $\frac{1}{n} q_{i}\left((n G)^{\circ}\right)=F_{i}$ for $i \in\{0,1, \ldots, s\}$, then $X$ carries a $Q$-polynomial association scheme.

Proof. Since $n G=\sum_{\alpha \in A^{\prime}(X)} \alpha A_{\alpha}$, we get

$$
F_{i}=\frac{1}{n} q_{i}\left((n G)^{\circ}\right)=\frac{1}{n} \sum_{\alpha \in A^{\prime}(X)} q_{i}(\alpha) A_{\alpha} \in \mathfrak{A}:=\operatorname{Span}_{\mathbb{R}}\left\{A_{\alpha} \mid \alpha \in A^{\prime}(X)\right\} .
$$

Comparing dimensions, we find $\mathfrak{A}=\operatorname{Span}_{\mathbb{R}}\left\{F_{i}\right\}_{i=0}^{s}$. Since $F_{0}, F_{1}, \ldots, F_{s}$ are orthogonal projections, it follows that $\mathfrak{A}$ is closed under the multiplication. Hence we proved that $\left(X,\left\{R_{\alpha}\right\}_{\alpha \in A^{\prime}(X)}\right)$ is an association scheme, where $R_{\alpha}$ is the relation whose adjacency matrix is $A_{\alpha}$.

Now $\left\{F_{i}\right\}_{i=0}^{s}$ are the primitive idempotents of $\mathfrak{A}$. Moreover $\left(X,\left\{R_{\alpha}\right\}_{\alpha \in A^{\prime}(X)}\right)$ is a $Q$-polynomial association scheme because of $F_{i}=\frac{1}{n} q_{i}\left((n G)^{\circ}\right)=\frac{1}{n} q_{i}\left(\left(n F_{1}\right)^{\circ}\right)$.

From Lemmas 3.7 and 3.8 we proved that a spherical 2-design which is an $s$ distance set and $S=s$ has the structure of a $Q$-polynomial scheme with respect to the idempotents $\left\{F_{i}\right\}_{i=0}^{s}$ if and only if $\frac{1}{n} q_{i}\left((n G)^{\circ}\right)=F_{i}$ for $i \in\{0,1, \ldots, s\}$.

\section{An EXCESS THEOREM FOR SPHERICAL 2-DESIGNS}

Recall that the spectral excess theorem implies that, for a graph $\Gamma=(X, E)$, the mean value of excesses $\left\{\left|\Gamma_{d}(x)\right|\right\}_{x \in X}$ is bounded above by using the predistance polynomial $p_{d}$ of degree $d$ and equality holds if and only if $\Gamma$ is distance-regular. In this section we give an excess theorem for a spherical 2-design $X$ which is an $s$-distance set and $S=s$. For $\boldsymbol{x} \in X$, put $m_{s}(\boldsymbol{x})=n\left(F_{s}\right)_{\boldsymbol{x}, \boldsymbol{x}}$, and $m_{s}(\boldsymbol{x})$ is called the excess of $\boldsymbol{x}$ in terms of $X$. The following theorem is the main theorem in this paper.

Theorem 4.1. Suppose a spherical 2-design $X$ is an $s$-distance set with $S=s$. Then the inequality

$$
\operatorname{tr} F_{s}=\frac{1}{n} \sum_{\boldsymbol{x} \in X} m_{s}(\boldsymbol{x}) \leq q_{s}(m)
$$

holds and equality is attained if and only if $X$ has the structure of a $Q$-polynomial association scheme with respect to the idempotents $\left\{F_{i}\right\}_{i=0}^{s}$.

In order to prove Theorem 4.1, we give some notation and a lemma. The set $\mathfrak{A}^{*}=\mathfrak{A}^{*}(X)=\left\{\frac{1}{n} p\left((n G)^{\circ}\right) \mid p \in \mathbb{R}[t]\right\}$ is a vector space of dimension $s+1$ and $\left\{J, G, \ldots, G^{\circ s}\right\}$ is a basis of $\mathfrak{A}^{*}$. Let $\mathfrak{D}^{*}=\mathfrak{D}^{*}(X)$ be the linear span of the set 
$\left\{F_{i}\right\}_{i=0}^{s}$. In our context, we will work with the vector space $\mathfrak{T}^{*}=\mathfrak{A}^{*}+\mathfrak{D}^{*}$. Note that $J, G$ and $I$ are matrices in $\mathfrak{A}^{*} \cap \mathfrak{D}^{*}$ since $I=\frac{1}{n} H\left((n G)^{\circ}\right) \in \mathfrak{A}^{*}$. Moreover, we have:

$$
F_{0}+F_{1}+\cdots+F_{s}=I=\frac{1}{n}\left(q_{0}\left((n G)^{\circ}\right)+q_{1}\left((n G)^{\circ}\right)+\cdots+q_{s}\left((n G)^{\circ}\right)\right) .
$$

Lemma 4.2. Suppose a spherical 2-design $X$ is an s-distance set with projection matrices $\left\{F_{i}\right\}_{i=0}^{s}$. Then $X$ has the structure of a $Q$-polynomial association scheme with respect to the idempotents $\left\{F_{i}\right\}_{i=0}^{s}$ if and only if $F_{s}=\frac{1}{n} q_{s}\left((n G)^{\circ}\right)$.

Proof. By Lemma 3.7, if $X$ has the structure of a $Q$-polynomial association scheme with respect to the idempotents $\left\{F_{i}\right\}_{i=0}^{s}$, then $F_{s}=\frac{1}{n} q_{s}\left((n G)^{\circ}\right)$. We prove that if $F_{s}=\frac{1}{n} q_{s}\left((n G)^{\circ}\right)$, then $X$ has the structure of a $Q$-polynomial association scheme with respect to the idempotents $\left\{F_{i}\right\}_{i=0}^{s}$. By Lemma 3.8, it suffices to show $F_{k}=$ $\frac{1}{n} q_{k}\left((n G)^{\circ}\right)$ for each $k \in\{2,3, \ldots, s-1\}$. First we check $F_{s-1}=\frac{1}{n} q_{s-1}\left((n G)^{\circ}\right)$. From (4.1) and the recurrence (3.2) for the predegree polynomials, we obtain the following two equalities:

$$
\begin{gathered}
F_{0}+F_{1}+\cdots+F_{s-1}=\frac{1}{n} q_{0}\left((n G)^{\circ}\right)+\frac{1}{n} q_{1}\left((n G)^{\circ}\right)+\cdots+\frac{1}{n} q_{s-1}\left((n G)^{\circ}\right), \\
(n G) \circ F_{s}=b_{s-1}^{*} \frac{1}{n} q_{s-1}\left((n G)^{\circ}\right)+a_{s}^{*} F_{s} .
\end{gathered}
$$

Then for $\varphi \in \operatorname{Harm}_{s}(X)$, we have

$$
\begin{aligned}
\left(\frac{1}{n} q_{s-1}\left((n G)^{\circ}\right) \varphi\right)(\boldsymbol{x}) & =\frac{1}{n} \sum_{\boldsymbol{y} \in X} \zeta_{\boldsymbol{x}}\left(q_{s-1}\right)(\boldsymbol{y}) \varphi(\boldsymbol{y}) \\
& =\left(\zeta_{\boldsymbol{x}}\left(q_{s-1}\right), \varphi\right)=0
\end{aligned}
$$

for every $\boldsymbol{x} \in X$, since $\zeta_{\boldsymbol{x}}\left(q_{s-1}\right)$ is in $\operatorname{Pol}_{s-1}(X)$. For $\psi \in \bigoplus_{i=0}^{s-2} \operatorname{Harm}_{i}(X)$, we have

$$
\begin{aligned}
\left((n G) \circ F_{s} \psi\right)(\boldsymbol{x}) & =\sum_{\boldsymbol{y} \in X}(\boldsymbol{x} \cdot \boldsymbol{y})\left(F_{s}\right)_{\boldsymbol{x}, \boldsymbol{y}} \psi(\boldsymbol{y}) \\
& =\sum_{\boldsymbol{y} \in X}\left(F_{s}\right)_{\boldsymbol{x}, \boldsymbol{y}} \zeta_{\boldsymbol{x}}(t)(\boldsymbol{y}) \psi(\boldsymbol{y}) \\
& =\left(F_{s} \cdot \zeta_{\boldsymbol{x}}(t) \psi\right)(\boldsymbol{x})=0
\end{aligned}
$$

for every $\boldsymbol{x} \in X$, since $\zeta_{\boldsymbol{x}}(t) \psi$ is in $\operatorname{Pol}_{s-1}(X)$. Thus, the equality (4.3) implies that

$$
\left(\frac{1}{n} q_{s-1}\left((n G)^{\circ}\right) \psi\right)(\boldsymbol{x})=0 .
$$

Hence, for every $f \in C(X)$, we have $\frac{1}{n} q_{s-1}\left((n G)^{\circ}\right) f \in \operatorname{Harm}_{s-1}(X)$. Multiplying both sides of (4.2) from the left by $F_{s-1}$, we have

$$
F_{s-1}=F_{s-1} \frac{1}{n} q_{s-1}\left((n G)^{\circ}\right),
$$

by Lemma 3.6. For $\chi \in \operatorname{Harm}_{s-1}(X)$, we put $\tilde{\chi}=\frac{1}{n} q_{s-1}\left((n G)^{\circ}\right) \chi$. Then, from (4.4), we have

$$
\begin{aligned}
\tilde{\chi} & =F_{s-1} \tilde{\chi}=F_{s-1} \frac{1}{n} q_{s-1}\left((n G)^{\circ}\right) \chi \\
& =F_{s-1} \chi=\chi .
\end{aligned}
$$

Thus, it follows that $\frac{1}{n} q_{s-1}\left((n G)^{\circ}\right)=F_{s-1}$. 
Let $2 \leq k \leq s-2$ and suppose now that $\frac{1}{n} q_{i}\left((n G)^{\circ}\right)=F_{i}$ for $s \geq i \geq k+1$. Then we have the following two equalities:

$$
\begin{gathered}
F_{0}+F_{1}+\cdots+F_{k}=\frac{1}{n} q_{0}\left((n G)^{\circ}\right)+\frac{1}{n} q_{1}\left((n G)^{\circ}\right)+\cdots+\frac{1}{n} q_{k}\left((n G)^{\circ}\right), \\
(n G) \circ F_{k+1}=b_{k}^{*} \frac{1}{n} q_{k}\left((n G)^{\circ}\right)+a_{k+1}^{*} F_{k+1}+c_{k+2}^{*} F_{k+2} .
\end{gathered}
$$

As before, from $\operatorname{deg} q_{k}=k$, we infer that $\frac{1}{n} q_{k}\left((n G)^{\circ}\right) \varphi=0$ for $\varphi \in \bigoplus_{i=k+1}^{s} \operatorname{Harm}_{i}(X)$. By the same reasoning as above, (4.6) yields $\frac{1}{n} q_{k}\left((n G)^{\circ}\right) \psi=0$ for $\psi \in \bigoplus_{i=0}^{k-1} \operatorname{Harm}_{i}(X)$. Hence, for any $f \in C(X)$, we have $\frac{1}{n} q_{k}\left((n G)^{\circ}\right) f \in \operatorname{Harm}_{k}(X)$. For $\chi \in \operatorname{Harm}_{k}(X)$, we can similarly prove $\frac{1}{n} q_{k}\left((n G)^{\circ}\right) \chi=\chi$. Then we have that $\frac{1}{n} q_{k}\left((n G)^{\circ}\right)=F_{k}$ which, by induction, proves the result.

We define an inner product in $M_{X}(\mathbb{R})$ by, for $R, S \in M_{X}(\mathbb{R})$,

$$
\langle R, S\rangle_{\mathrm{m}}=\operatorname{tr}\left({ }^{t} R S\right) .
$$

Observe that, for $p, q \in \mathbb{R}[t]$, we have

$$
\left\langle\frac{1}{n} p\left((n G)^{\circ}\right), \frac{1}{n} q\left((n G)^{\circ}\right)\right\rangle_{\mathrm{m}}=\frac{1}{n^{2}} \sum_{\alpha \in A^{\prime}(X)} \kappa_{\alpha} p(\alpha) q(\alpha)=\langle p, q\rangle .
$$

Consider the Euclidean space $\mathfrak{T}^{*}$ with the inner product (4.7) and the orthogonal projection $\mathfrak{T}^{*} \rightarrow \mathfrak{A}^{*}$ denoted by $S \mapsto \tilde{S}$. Using in $\mathfrak{A}^{*}$ the orthogonal basis $\left\{\frac{1}{n} q_{i}\left((n G)^{\circ}\right)\right\}_{i=0}^{s}$ of the predegree polynomials of $X$, this projection can be expressed as

$$
\tilde{S}=\sum_{i=0}^{s} \frac{\left\langle S, q_{i}\left((n G)^{\circ}\right)\right\rangle_{\mathrm{m}}}{n^{2} q_{i}(m)} q_{i}\left((n G)^{\circ}\right) .
$$

We put $\mu=\frac{1}{n} \sum_{\boldsymbol{x} \in X} m_{s}(\boldsymbol{x})$, for short.

Proof of Theorem 4.1. By Lemma 3.6 and since $\left\langle F_{s}, F_{s}\right\rangle_{\mathrm{m}}=\left\langle F_{s}, I\right\rangle_{\mathrm{m}}=\operatorname{tr}\left(F_{s}\right)=$ $\mu$, the projection of $F_{s}$ onto $\mathfrak{A}^{*}$ is

$$
\begin{aligned}
\tilde{F}_{s} & =\sum_{i=0}^{s} \frac{\left\langle F_{s}, q_{i}\left((n G)^{\circ}\right)\right\rangle_{\mathrm{m}}}{n^{2} q_{i}(m)} q_{i}\left((n G)^{\circ}\right)=\frac{\left\langle F_{s}, q_{s}\left((n G)^{\circ}\right)\right\rangle_{\mathrm{m}}}{n^{2} q_{s}(m)} q_{s}\left((n G)^{\circ}\right) \\
& =\frac{\left\langle F_{s}, H\left((n G)^{\circ}\right)\right\rangle_{\mathrm{m}}}{n^{2} q_{s}(m)} q_{s}\left((n G)^{\circ}\right)=\frac{\left\langle F_{s}, I\right\rangle_{\mathrm{m}}}{n q_{s}(m)} q_{s}\left((n G)^{\circ}\right) \\
& =\frac{\mu}{n q_{s}(m)} q_{s}\left((n G)^{\circ}\right) .
\end{aligned}
$$

Hence we get

$$
0 \leq\left\langle F_{s}, F_{s}\right\rangle_{\mathrm{m}}-\left\langle\tilde{F}_{s}, \tilde{F}_{s}\right\rangle_{\mathrm{m}}=\mu-\frac{\mu^{2}}{q_{s}(m)}=\mu\left(1-\frac{\mu}{q_{s}(m)}\right) .
$$

Since $\mu=\operatorname{tr}\left(F_{s}\right)=\operatorname{rank}\left(F_{s}\right)>0$, this implies the desired inequality. Moreover the equality is attained if and only $F_{s}=\tilde{F}_{s}=\frac{1}{n} q_{s}\left((n G)^{\circ}\right)$. By Lemma 4.2 the desired result follows. 
Acknowledgments. The author would like to thank Eiichi Bannai, Edwin van Dam, Tatsuro Ito, Jack Koolen, William J. Martin, Akihiro Munemasa and Hajime Tanaka for useful discussions and comments. Part of this work was carried out while the author was visiting the Mathematisches Forschungsinstitut Oberwolfach. The institute kindly offered the stay while his University was affected by the 2011 Tohoku earthquake. The author is grateful to the MFO and its staffs for the offer, financial support and warm hospitality. The author also would like to thank the Leibniz Association for travel support. The author is supported by the fellowship of the Japan Society for the Promotion of Science.

\section{REFERENCES}

[1] Ei. Bannai and Et. Bannai. A survey on spherical designs and algebraic combinatorics on spheres. European J. Combin., 30(6):1392-1425, 2009.

[2] E. Bannai and T. Ito. Algebraic combinatorics. I. The Benjamin/Cummings Publishing Co. Inc., Menlo Park, CA, 1984.

[3] A. E. Brouwer, A. M. Cohen, and A. Neumaier. Distance-regular graphs, volume 18 of Ergebnisse der Mathematik und ihrer Grenzgebiete (3) [Results in Mathematics and Related Areas (3)]. Springer-Verlag, Berlin, 1989.

[4] P. J. Cameron, J. M. Goethals, and J. J. Seidel. The Krein condition, spherical designs, Norton algebras and permutation groups. Nederl. Akad. Wetensch. Indag. Math., 40(2):196206, 1978.

[5] H. S. M. Coxeter. Regular Polytopes, 3rd Edition. Dover, 1973.

[6] D. M. Cvetković, M. Doob, and H. Sachs. Spectra of graphs, volume 87 of Pure and Applied Mathematics Theory and application. Academic Press Inc. [Harcourt Brace Jovanovich Publishers], New York, 1980.

[7] E. R. van Dam. The spectral excess theorem for distance-regular graphs: a global (over)view. Electron. J. Combin., 15(1):\#R 129 1-10, 2008.

[8] P. Delsarte, J. M. Goethals, and J. J. Seidel. Spherical codes and designs. Geometriae Dedicata, 6(3):363-388, 1977.

[9] M. A. Fiol and E. Garriga. From local adjacency polynomials to locally pseudo-distanceregular graphs. J. Combin. Theory Ser. B, 71(2):162-183, 1997.

[10] M. A. Fiol, S. Gago, and E. Garriga. A simple proof of the spectral excess theorem for distance-regular graphs. Linear Algebra Appl., 432(9):2418-2422, 2010.

[11] C. D. Godsil. Polynomial spaces. Discrete Math., 73(1)-(2):71-88, 1989.

[12] C. D. Godsil. Algebraic combinatorics, Chapman \& Hall, New York, 1993.

[13] H. Kurihara and H. Nozaki. A characterization of $Q$-polynomial association schemes. J. Combin. Theory Ser. A, 119(1): 57-62, 2012.

Mathematical Institute, Tohoku University, Aramaki-Aza-Aoba 6-3, Aoba-ku, Sendai 980-8578, JAPAN

E-mail address: sa9d05@math.tohoku.ac.jp 